\title{
3D Sphere-like Nickel Tungstate Catalyst for Destroying of Organophosphate Pesticide Methyl Parathion
}

\author{
J. Vinoth Kumar, M. Arunpadian, D. Sivaganesh, E. R. Nagarajan
}

\begin{abstract}
Recently, the appearance of pesticide residues into the environment poses serious risks to the living organisms. In this view, we explored a novel and superior photocatalyst based on nanostructured sphere-like nickel tungstate $\left(\mathrm{NiWO}_{4} \mathrm{NSs}\right)$ for the degradation of organophosphate pesticide methyl parathion (MP) under visible light irradiation, for the first time. The $\mathrm{NiWO}_{4} \mathrm{NSs}$ were tailored through simple precipitation technique and confirmed by several spectroscopic techniques. Fascinatingly, the $\mathrm{NiWO}_{4} \mathrm{NSs}$ portrayed superior photodegradation performances towards MP degradation with superior stability.
\end{abstract}

Keywords: Photocatalyst, Pesticides, Methyl Parathion, Nanostructures.

\section{INTRODUCTION}

As we all are known that, the food, fruits, vegetables, grains and wheat's are an important and more basic sources in order to live the both human beings and animals. At the meantime, firing growth of industrialization, urbanization and the decrement of crop producing lands, results in huge food shortage. To ensure the above dilemma, merchants were adulterated the food products in order to increase the quantity. On the other side, famers are mostly sprayed the pesticides in crops to earn the more profit in very short period of time. Among those pesticides, organophosphorous phosphorous compounds, especially, methyl parathion has been broadly used pesticide which induces severe heart problem, skin diseases and various unknown diseases for both animal and humans [1,2]. For these troubles, totally detoxification of MP into the aqueous and land areas is crucial role to the researchers. For degradation system, photocatalysis method is most adoptable route [3]. During

Revised Manuscript Received on December 15, 2019

* Correspondence Author

E. R. Nagarajan*, Department of Chemistry, Nanomaterials Laboratory, International Research Centre, Kalasalingam Academy of Research and Education, Krishnankoil-626126, Tamil Nadu, India.

Email: nagarajanklu@gmail.com.

J. Vinoth Kumar, Department of Chemistry, Nanomaterials Laboratory, International Research Centre, Kalasalingam Academy of Research and Education, Krishnankoil-626126.Tamil Nadu, India.

Email: vinothchem7@gmail.com

D. Sivaganesh, Department of Physics, International Research Centre, Kalasalingam Academy of Research and Education, Krishnankoil- 626126, Tamil Nadu, India.

Email: ganesh.siva650@gmail.com

M. Arunpandian, Department of Chemistry, Nanomaterials Laboratory, International Research Centre, Kalasalingam Academy of Research and Education, Krishnankoil-626126, Tamil Nadu, India.

Email: arunpandiantt126@gmail.com the photocatalytic reaction make hydroxyl radicals $(\bullet \mathrm{OH}$.) and superoxide radicals $(\mathrm{O} 2 \bullet-)$ with more superior oxidizing ability which destroy the all the organic pollutants into carbon dioxide and water molecules [4]. In particularly, heterogeneous photocatalysis route is the most superior and effective strategy.

Mixed transition metal oxides have been attracted superior interest in numerous fundamental and technological applications when compared to single metal oxides [5]. Among the transition metal oxides, nickel oxides $(\mathrm{NiO})$ and tungsten trioxide $\left(\mathrm{WO}_{3}\right)$ have been widely in energy and environmental applications owing to their good physicochemical nature [6]. Enthused by advantages of binary metal oxides, several researchers have been fabricated nickel tungstate $\left(\mathrm{NiWO}_{4}\right)$ nanoparticles by using several techniques including precipitation, sonication, hydrothermal, ball milling, chemical vapor deposition and solvothermal routes. It seems that the simple co-precipitation method has several advantages such as facile, cost-effective, quick, low temperature synthesis and does not require special conditions [7,8]. Therefore, synthesis of $\mathrm{NiWO}_{4}$ via simple co-precipitation may enrich the catalytic properties.

In this report, we synthesized 3D sphere-like $\mathrm{NiWO}_{4}$ nanostructures by precipitation route and applied as a photocatalyst for the removal of hazardous MP aqueous suspension under visible light exposure. To the best of our mind, this is the first report for the design of $\mathrm{NiWO}_{4} \mathrm{NSs}$ for the MP degradation.

\section{EXPERIMENTAL}

\section{A. Materials}

Nickel chloride hexahydrate $\left(\mathrm{NiCl}_{2} \cdot 6 \mathrm{H}_{2} \mathrm{O}\right)$, sodium tungstate dihydrate $\left(\mathrm{Na}_{2} \mathrm{WO}_{4} \cdot 2 \mathrm{H}_{2} \mathrm{O}\right)$ and methyl parathion chemicals were bought from Alfa Aesar Company, Madurai, Tamil Nadu, India.

\section{B. Synthesis of $\mathrm{NiWO}_{4} \mathrm{NSs}$}

In this procedure, $0.05 \mathrm{M} \mathrm{NiCl} 2 \cdot 6 \mathrm{H}_{2} \mathrm{O}$ and $0.1 \mathrm{M}$ $\mathrm{Na}_{2} \mathrm{WO}_{4} \cdot 2 \mathrm{H}_{2} \mathrm{O}$ were dissolved in $80 \mathrm{~mL}$ double distilled water under vigorous magnetic stirring for $1 \mathrm{~h}$. Consequently, the obtained green color particles were thoroughly washed with water/ethanol and acetone for five times. The washed particles were dried at $80^{\circ} \mathrm{C}$ for $10 \mathrm{~h}$. In final, the dried green products were 
calcined air atmosphere at $450^{\circ} \mathrm{C}$ for $4 \mathrm{~h}$.

\section{RESULTS AND DISCUSSION}

Powder X-ray diffraction (PXRD) analysis was used to identify the crystalline structure of the material. As from the Fig. 1, the observed major diffraction peaks at $2 \theta=19.28^{\circ}$, $23.97^{\circ}, 24.91^{\circ}, 30.96^{\circ}, 36.62^{\circ}, 41.72^{\circ}$ and $54.68^{\circ}$ were ascribed to the (100), (011), (110), (111), (002), (121) and (202) hkl planes of monoclinic phase structure of $\mathrm{NiWO}_{4}$. The results are good consistence with their JCPDS No. 72-0480. From the XRD pattern, there was no extra diffraction peaks related to the $\mathrm{NiO}$ or $\mathrm{WO}_{3}$ detected, illustrated that the high phase purity. In addition, the well sharp further demonstrated the very good crystallinity of the $\mathrm{NiWO}_{4}$.

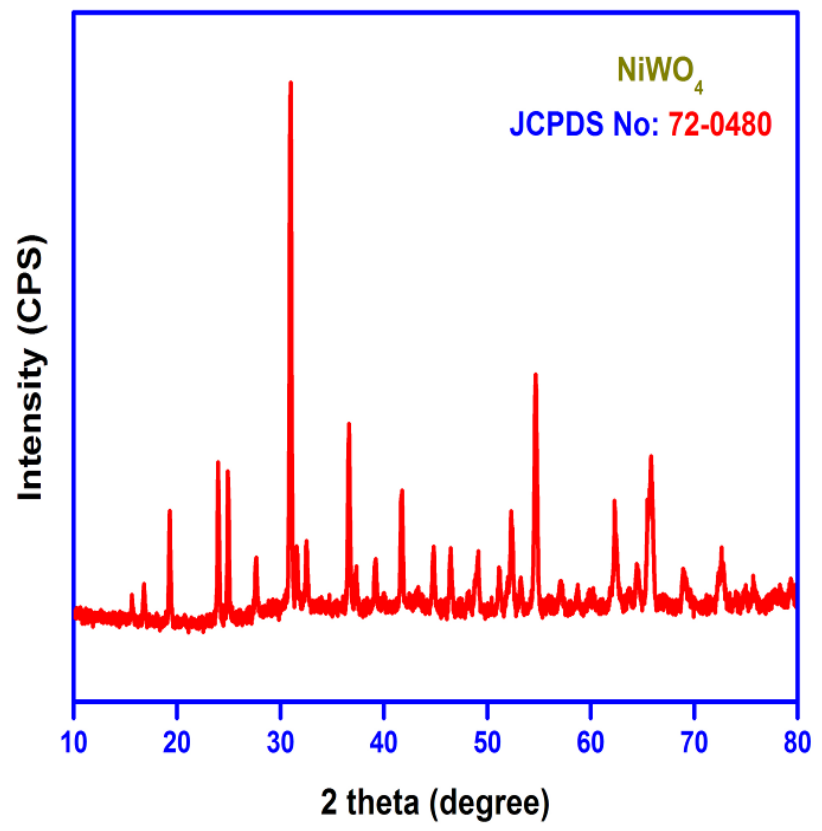

Fig. 1. XRD pattern of $\mathrm{NiWO}_{4}$.

The topography of the $\mathrm{NiWO}_{4}$ was visualized by SEM studies. The dissimilar magnification SEM images (Fig. 2 $(A \& B)$ ) clearly portrayed that the sphere-like structure with the smooth surfaces. The obtained 3D sphere-like could be produced of small two dimensional sheets with the average diameter is approximately to be $60 \mathrm{~nm}$.

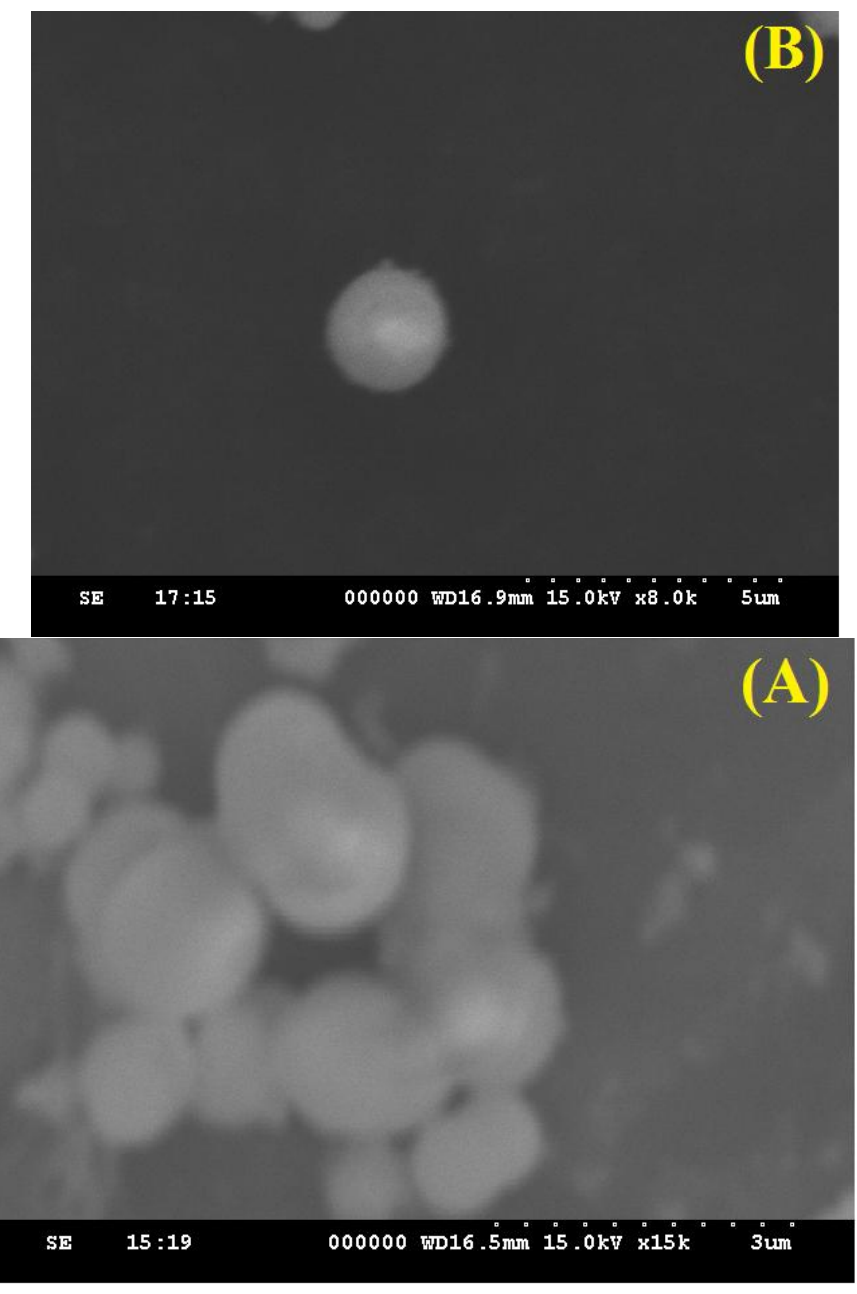

Fig. 2. (A\&B) Different magnification SEM images of $\mathrm{NiWO}_{4}$.

The elements which present in the surface of the $\mathrm{NiWO}_{4}$ NSs was observed by EDS analysis and represented in Fig. 3 It revealed that the attendance of $\mathrm{Ni}, \mathrm{W}$ and $\mathrm{O}$ elements without impurities.

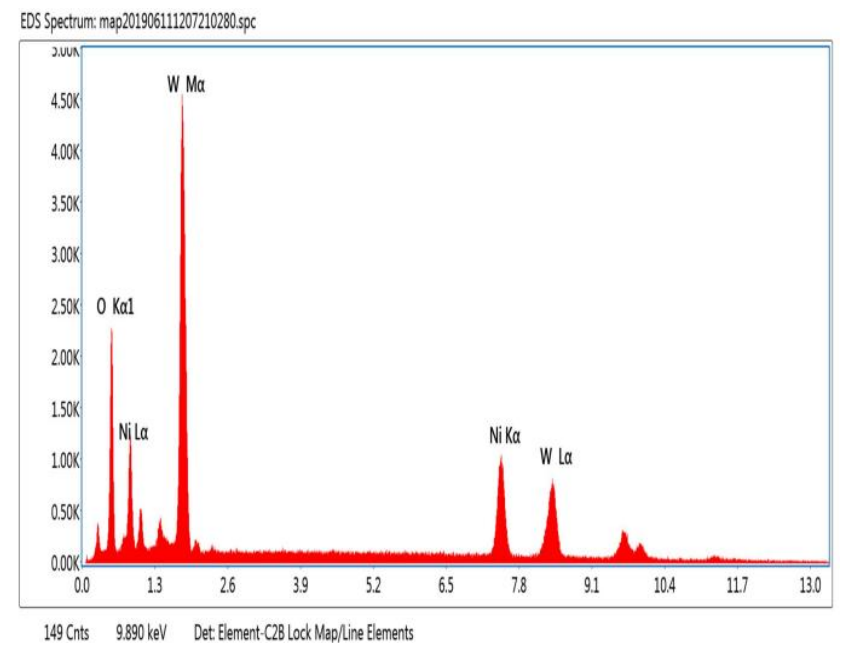

Fig. 3. EDX spectrum of $\mathrm{NiWO}_{4}$.

The colored images in Fig. 4 (B-D) clearly confirmed that the uniform distribution of $\mathrm{Ni}$, 
$\mathrm{W}$ and $\mathrm{O}$ elements in the overall scanning region in Fig. $4 \mathrm{~A}$.
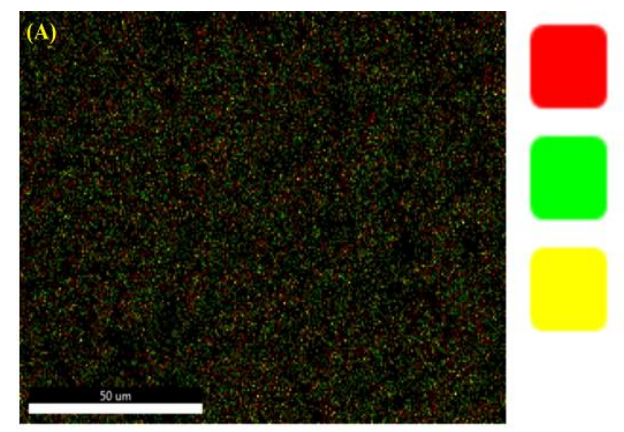

\section{$34 \%$ O K}

$36 \%$ NiK

$30 \%$ W L
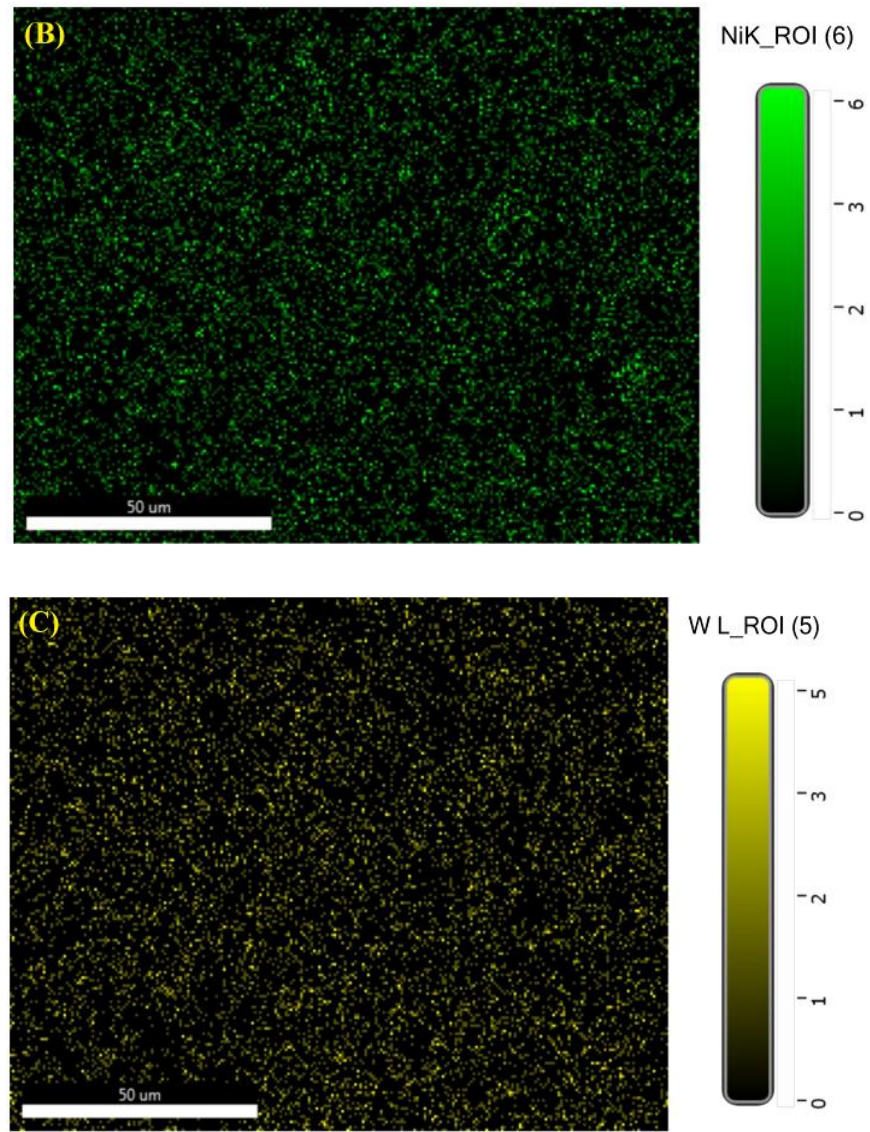

W L_ROI (5)

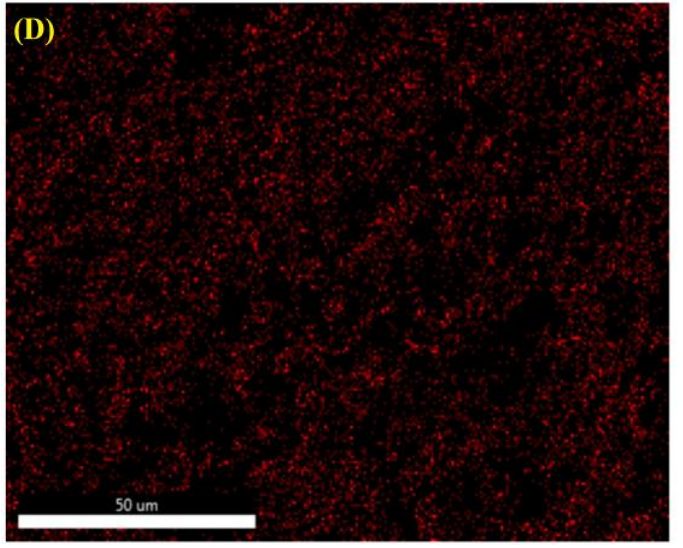

OK_ROI (6)

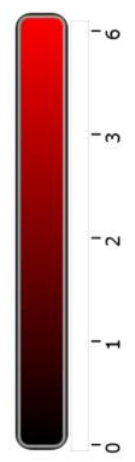

Fig. 4. (A-D) EDX elemental mapping of spectrum of $\mathrm{NiWO}_{4}$.

\section{A. Photocatalytic activity}

The photocatalytic efficacy of $\mathrm{NiWO}_{4} \mathrm{NSs}$ (catalyst dosage $=50 \mathrm{mg} / \mathrm{mL}$ ) were tested in opposition to the degradation of MP pesticide aqueous suspension $(5 \mathrm{mg} / \mathrm{L})$ under visible light exposure (Solution $\mathrm{pH}=5.5$ ). Fig. 5 portrayed the major absorption intensity peak of MP at 278 $\mathrm{nm}$ reduces gradually and the peak was disappeared after 70 min of light exposure. As from the Fig. 5, no other intermediate peaks were obtained, illustrated that the successful degradation of MP in the presence of $\mathrm{NiWO}_{4} \mathrm{NSs}$.

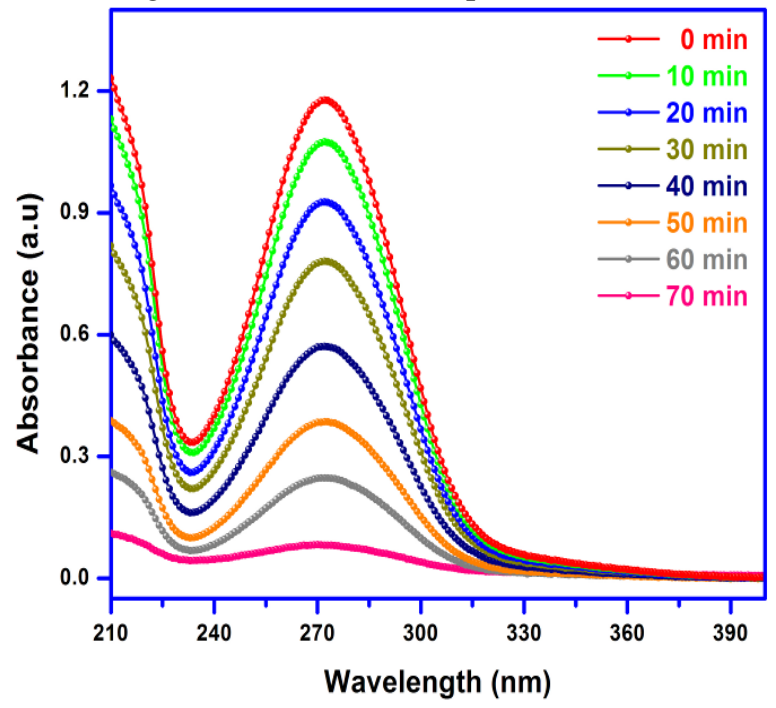

Fig. 5. Time-temporal UV-visible spectrum of MP photodegradation by $\mathrm{NiWO}_{4}$.

Based on the previous work, we have included the possible degradation pathway of MP in Fig.6.

(a)

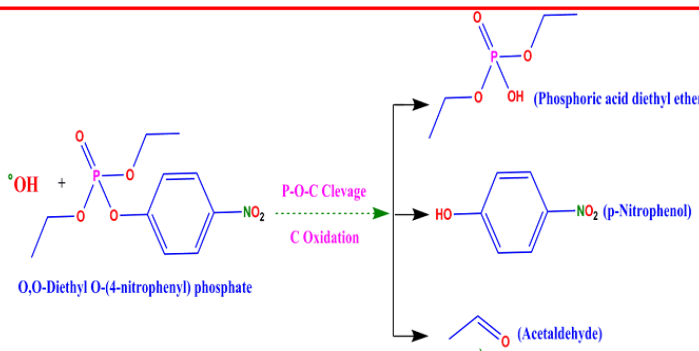

(b)

(Phosphoric acid diethyl ether)

(c)

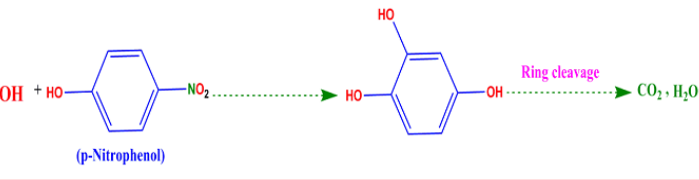


Fig. 6. Photodegradation pathway of MP.

\section{CONCLUSION}

In summary, an efficient $\mathrm{NiWO}_{4} \mathrm{NSs}$ were successfully synthesized by simple precipitation route and employed as a photocatalyst for the degradation of MP pesticide very effectively. These studies fascinate the superior idea for the nanomaterials synthesis for industrial aspects.

\section{ACKNOWLEDGMENT}

We are grateful to thank the Kalasalingam Academy of Research and Education, Tamil Nadu, India for providing research facilities.

\section{REFERENCES}

1. J. V. Kumar, R. Karthik, Shen-Ming Chen, K. Natarajan, K. Chelladurai, C. C. Yang, V. Muthuraj, "3D Flower-Like Gadolinium Molybdate Catalyst for Efficient Detection and Degradation of Organophosphate Pesticide (Fenitrothion)," ACS Appl. Mater. Interfaces, 10 (2018) $15652-15664$

2. J. V. Kumar, R. Karthik, Shen-Ming Chen, V. Muthuraj, C. Karuppiah, "Fabrication of potato-like silver molybdate microstructures for photocatalytic degradation of chronic toxicity ciprofloxacin and highly selective electrochemical detection of H2O2," Sci. Rep., 6 (2017) 34149.

3. R. Karthik, J. V. Kumar, S. M. Chen, P. S. Kumar, V. Selvam, V. Muthuraj, "A selective electrochemical sensor for caffeic acid and photocatalyst for metronidazole drug pollutant - A dual role by rod-like SrV2O6," Sci. Rep., 7 (2017) 7254.

4. Y. P. Bhoi, A. K. Nayak, S. K. Gouda, B. G. Mishra, "Photocatalytic mineralization of carbendazim pesticide by a visible light active novel type-II Bi2S3/BiFeO3 heterojunction photocatalyst”. Catal. Commun. 114 (2018) 114-119.

5. D. Jornet, M. A. Castillo, M. C. Sabater, R. Tormos, M. A. Miranda, "Photodegradation of carbendazim sensitized by aromatic ketones" J. Photochem. Photobiol. A. 256 (2013) 36-41.

6. K. Adib, M. Rahimi-Nasrabadi, Z. Rezvani, S. M. Pourmortazavi, F. Ahmadi, H. R. Naderi, M. R. Ganjali, "Facile chemical synthesis of cobalt tungstates nanoparticles as high performance supercapacitor”J. Mater. Sci. Mater. Electron. 227, (2016) 4541-4550.

7. J. Vinoth Kumar, R. Karthik, S. M. Chen, P. Balasubramanian, V. Muthuraj, V. Selvam, "A novel cerium tungstate nanosheets modified electrode for the effective electrochemical detection of carcinogenic nitrite ions" Electroanalysis, 29 (2017) 2385-2394.

8. P. Sundaresan, A. Krishnapandi, S. M. Chen, "Design and investigation of ytterbium tungstate nanoparticles: An efficient catalyst for the sensitive and selective electrochemical detection of antipsychotic drug chlorpromazine" J. Taiwan. Inst. E. 96 (2019) 509-519.

\section{AUTHORS PROFILE}

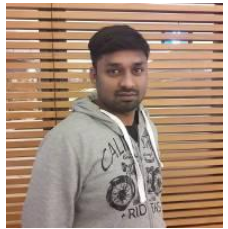

Dr. J. Vinoth kumar is a Post-doctoral fellow at Kalasalingam university, Virudhunagar, India. He got his Ph.D Degree from Madurai Kamaraj University, Madurai, India. $\mathrm{He}$ is interested in synthesis of nanomaterials for photocatalysis and electrocatalysis applications.

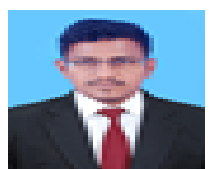

D. Sivaganesh is a Ph.D student at Kalasalingam University, India. His research interest is in structural and photoluminescence properties of phosphor materials.

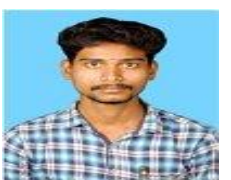

M. Arunpandian is a $\mathrm{Ph} . \mathrm{D}$ student at Kalasalingam University, India. His research interest is in photocatalytic properties of rare earth attached metal oxides composite materials.

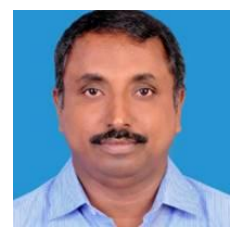

E.R. Nagarajan works as Associate Professor, Department of Chemistry, Kalasalingam Academy of Research and Education, Krishnankoil, Tamil Nadu, India. He received his Ph.D degree at Anna University, Chennai, India. With a research in the applications of polymeric materials, co-ordination compounds, photocatalysis and electrochemical applications. 